\title{
70 EXTERNAL CAUSES OF INJURIES IN TEHRAN, IRAN: A HOUSEHOLD SURVEY
}

doi:10.1136/injuryprev-2012-040590w.70

${ }^{1} \mathrm{M}$ Zangi, ${ }^{1} \mathrm{R}$ Mohammadi, ${ }^{2} \mathrm{~S}$ Saadat. ${ }^{1}$ Karolinska institutet, Sweden; ${ }^{2}$ Sina Hospital, Iran

Background Injuries continue to be significant health problems and leading causes of death and disability in many populations.

Aims A household survey was conducted to determine the external causes of injuries among people living in Tehran, Iran.

Methods Using cluster random sampling approach, a household survey was conducted in April 2007, seeking information about external causes and severity of injuries occurred within families in past Persian year. External causes were classified according to ICD-10 and analysed using SPSS 16.

Results Out of 9173 household participants, 765 ones (8.3\%) had injuries with male to female ratio of $0.54: 1$ during the past Persian year. Injuries occurred mostly due to 'exposure to the inanimate mechanical forces', while the second and third causes were 'contact with heat or hot substances' and 'falls', respectively. When it comes to required attention injuries, the incidence rate was 175.5 per 10000 person per year with male to female ratio of $2.37: 1$ and mostly associated with 'falls' and 'transport accidents'. Exposure to inanimate mechanical forces and also burns mostly occurred among middle aged women at home and leaded to open wounds and superficial burns respectively. Young boys were more vulnerable to falls which mostly took place at home and sport places and frequently resulted in superficial injuries and fractures. Traffic accidents mostly took place among middle aged men and leaded to fractures and superficial injuries.

Contribution to the Field The priority in injury prevention programme should be given based on common external causes and to traffic and home injuries. 